\begin{tabular}{|l|l|l|l|l|l|}
\hline Revista Clío América & ISSN: 1909-941X & Vol. 10 & No. 20 & Julio - Diciembre de 2016 & 186 - 193 \\
\hline \multicolumn{6}{|c|}{ DOI: http://dx.doi.org/10.21676/23897848.1876 }
\end{tabular}

\title{
El ciclo cliente proveedor y la familia de normas ISO 9000
}

\author{
The ISO standards and the cycle client provider
}

Resumen: Los directivos actuales se enfrentan a una situación relativamente nueva: los clientes están cada día más informados y han aprendido a exigir mejor calidad, mayor variedad, mayor rapidez en la respuesta y precios acorde a sus expectativas. De ahí la importancia de controlar el comportamiento de la relación producto-insumo.

Las condiciones en que se comercializan los productos y servicios cambian a causa de un conjunto de fenómenos que caracterizan al mercado actual.

Se resumen en este trabajo las consideraciones sobre aspectos relacionados con la comprensión del enfoque de procesos de la familia de normas ISO, en contraste con el impacto que ejercen en el desempeño empresarial, fundamentalmente en América Latina.

La metodología usada ha sido la revisión documental, el análisis y la síntesis a través de la contrastación con experiencias empíricas del autor.

Palabras clave: enfoque por procesos, tecnología, calidad

JEL: M1

\begin{abstract}
Current managers are facing a relatively new situation: clients are every day more informed, and they have learned to demand better quality, more variety, faster response, and prices according to your expectations. Hence the importance of controlling the behavior of the relation input - product. The conditions in which products and services are marketed change, because of a set of phenomena that characterize the current market. Considerations on issues related to the understanding of the process approach of the family of ISO standards, in contrast to the impact they have on business performance, primarily in Latin America are summarized in this paper. The methodology used has been the document review, analysis, and synthesis via comparison with empirical experiences of the author.
\end{abstract}

Keywords: approach by processes, technology, quality
María Eugenia Palomeque-Solano Master en Auditoria y Contabilidad, Universidad Técnica de Machala, Ecuador. Email:mpalomeque@utmachala.edu.ec

Tipología: Artículo de Reflexión Fecha de Recibido: Mayo 10 de 2016 Fecha de Aceptación: Agosto 01 de 2016

Para citar este artículo: Palomeque, S. M. (2016). El ciclo cliente proveedor y la familia de normas ISO 9000.

Clío América, 10 (20), pp. 186 - 193 


\section{Introducción}

El cada vez más rápido avance de la tecnología facilita y abarata los procesos productivos y de servicios, pero complejiza su gerencia. Los nuevos materiales influyen en los procesos: abaratan los costos, disminuyen los tiempos de elaboración, simplifican las operaciones tecnológicas, por una parte y cambian los procesos de reciclaje o eliminación de desechos. Surgen nuevos productos o servicios casi a diario y con ellos, nuevas necesidades de los clientes, que marchan a tono con esos avances tecnológicos.

Con el aumento de la velocidad de cambio tecnológico disminuye la vida útil económica de productos y tecnologías. Hasta los años 50 del pasado siglo, el criterio para decidir si un equipo debía o no sustituirse en un proceso, era la relación entre el valor de lo que producía, y lo gastado en su producción. Como los gastos de mantenimiento tienen un comportamiento creciente en función del tiempo de explotación, la curva de gastos de mantenimiento decidía el momento en que un equipo era sustituido por uno nuevo. Como la velocidad de cambio tecnológico no era significativa, las sustituciones eran puntuales. El cambio de una máquina por otra nueva, no significaba que la anterior o posterior en el proceso productivo tuviera que ser cambiada. En síntesis, el equipamiento era producido para que durara un buen tiempo, y el desgaste físico era el criterio decisorio para las reposiciones (Alford, Bangs \& Hagemann, 1953, p.843).

A partir de la segunda década del siglo XX han sido las tecnologías de procesos las que han ido cambiando. Las sustituciones puntuales de equipamientos se han convertido en reposiciones totales de líneas de producción completas. El equipamiento se produce con altos estándares de fiabilidad, para un período definido de tiempo de explotación que los fabricantes cuidan mucho de declarar. La producción de componentes aislados de reposición para los equipos es cada vez menor $y$, a cambio, los gastos de mantenimiento crecen notablemente a costa de los elementos del costo vinculados al trabajo vivo. Una parte considerable del equipamiento productivo se adquiere incluyendo el mantenimiento especializado, brindado por el fabricante. Muchos leasing incluyen el recambio del equipo por otro de tecno- logía más avanzada, con tal de mantener al cliente cautivo. El cambio de suministrador resulta fácil, pues no hay gran lealtad de clientes y mantenerlo, brindándole un buen servicio, garantiza sobrevivir en un mercado cada vez más competitivo y globalizado.

En los años 90 del siglo XX se hizo popular el término "aldea global" debido a la disminución acelerada de los gastos en comunicación, que incidió en la disminución de los gastos de transporte. La competencia se internacionaliza, deja de ser regional. El desarrollo de las tecnologías de la informática y las comunicaciones tienen en ello una significativa participación.

A finales del siglo pasado también ocurre una disminución de las medidas proteccionistas. Se fortalecen zonas de libre comercio, se firman tratados y convenios internacionales o regionales, que contribuyen al auge del comercio. Simultáneamente, se establecen requisitos normativos rigurosos para acceder a mercados importantes, lo que exacerba las diferencias por el acceso a tecnologías costosas. Entre las exigencias normativas se destacan las de la Organización Internacional de Normalización (ISO) (ISO/IEC 20000-3:2012, 2012) (IS0, 2015), vinculadas originalmente a la calidad, y extendidas a la protección ambiental, la seguridad y salud, y a la integración sistémica del marco normativo.

Estas tendencias implican la disminución de la influencia de las economías de escala y la necesidad de desarrollar nuevos productos y servicios, con un reforzamiento de la función de Investigación y Desarrollo (I\&D) disminuyen las producciones contra almacén, se hacen más complejas las operaciones de predicción y las inversiones en prevención de fallas.

Por ello las organizaciones se ven obligadas a mejorar las comunicaciones, estrechar las relaciones con los clientes, aumentar las inversiones en I\&D, disminuir su tamaño, que permita aumentar la flexibilidad de respuesta, convirtiendo a la organización en una entidad con capacidad para aprender.

Este ensayo pretende argumentar que el paso del enfoque funcional tradicional de la actividad empresarial a uno por procesos es paulatino, y que exige la comprensión de los sistemas productivos particulares. 


\section{Operaciones y procesos}

Se llama gestión de operaciones a la coordinación sistematizada de los procesos organizacionales, para convertir la necesidad de un cliente en una solución integral; que tenga la calidad que el cliente esperaba, que sea la cantidad convenida, que se entregue en el plazo establecido, a un costo ventajoso; tanto para la organización que ofrece la solución, como para el cliente que la recibe (Castillo Coto, 2015, 2016).

En esta definición hay algunos aspectos interesantes:

- Se habla de coordinación sistematizada en lugar de los tradicionales pasos de planeación, organización, mando y control,

- Se habla de necesidad expresa del cliente, y no de demanda,

- No se trata de productos o servicios, sino de soluciones integrales,

- Se definen los principales aspectos que permiten al cliente seleccionar o no un suministrador,

- Se habla de un costo ventajoso para ambas partes, pues la ventaja de costo para la organización suministradora significa su supervivencia, y para la receptora del producto/servicio, su permanencia como cliente de la primera.

El enfoque de procesos de la familia de normas ISO impone una concepción cíclica de las relaciones cliente-proveedor, en lugar de la percepción lineal del enfoque funcional (Andreu, 2013). Este ciclo comienza y termina en el cliente, como se detalla en la Figura No. 1 mediante de la representación de los flujos Informativo, Material y Financiero.

Como la velocidad del cambio tecnológico es cada vez mayor, el cliente expresa una necesidad que la organización suministradora estaría en condiciones de satisfacer, no necesariamente con un producto determinado.

Marketing que actúa como una estructura de interfaz entre la organización y el entorno detecta esa expresión del cliente y la hace llegar a I\&D. Es esta función la que va a convertir la necesidad del cliente en una solución y va a trasladar, a su vez, la orden a las estructuras de Producción.

Producción será quien determine los insumos necesarios para la elaboración del producto o la prestación del servicio que espera el cliente, y pase la información a Aprovisionamiento, que enviará la información del pedido a los Proveedores.

En esta primera fase el flujo es informativo. Se convierte en material a partir de que los Proveedores hagan el envío correspondiente al pedido efectuado por Aprovisionamiento, quien lo entrega a Producción que elabora el producto deseado por el cliente y lo entrega a Distribución/Ventas que lo hace llegar al Cliente.

Cuando la entrega ha sido efectuada, culmina el flujo material y comienzan dos flujos de reversa: uno financiero porque el Cliente efectúa el pago del servicio o producto, que permite que Aprovisionamiento pueda efectuar los pagos a los Proveedores y otro informativo, pues Marketing va a comprobar si el Cliente está satisfecho con el producto o servicio y en función de la información obtenida va a retroalimentar a la estructura correspondiente.

Obsérvese que no se han referido estructuras funcionales de la empresa sino funciones, fases de un proceso holísticamente integrado. Es esta visión de ciclo continuo la que justamente diferencia el actuar de la empresa en el ámbito de este marco normativo.

\section{Elementos de Atención}

Con la difusión de la familia de normas ISO (International Organization for Standardization) (ISO, 2015) se ha difundido el enfoque de gestión de procesos y, con ello, una manera más holística de analizar sistémicamente las organizaciones. Quiere decir que pueden representarse los elementos que centran su atención en la gestión de operaciones al usar para ello el enfoque SIPOC (de los términos en inglés: Suppliers, Inputs, Processes, Outputs y Clients) que utilizan las Normas ISO, como se muestra en la Figura No. 2. 


\section{Figura 1.}

\section{Relación cliente - proveedor}

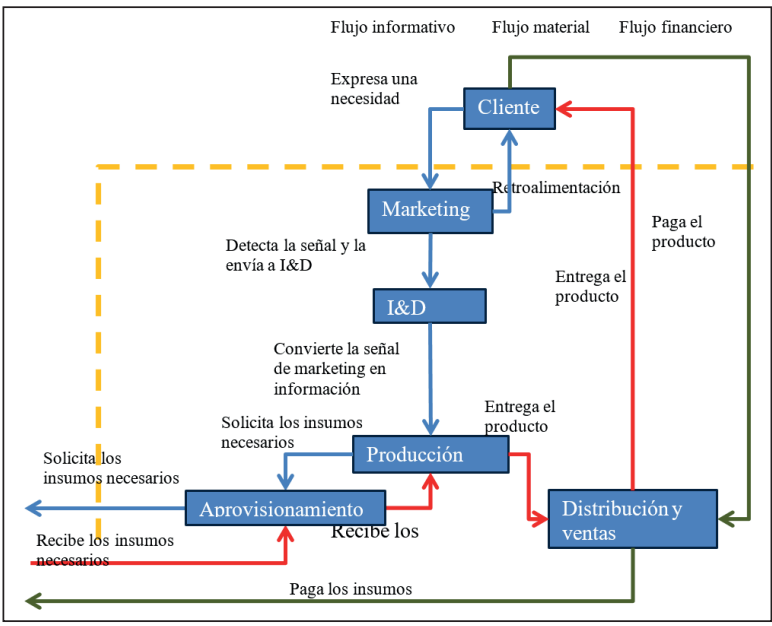

Fuente: Elaboración propia

A continuación, se analizan los aspectos que tienen relación con la gestión de operaciones.

\section{Insumos del sistema organizacional}

El elemento determinante para todo proceso de agregación de valor es el talento humano. Sin hombres no hay proceso productivo, ni de prestación de servicios. Por muy avanzada que sea la tecnología, es el trabajo humano su creador. El conocimiento de los hombres es, por tanto, el factor determinante del éxito de cualquier proceso. Ese conocimiento se manifiesta en competencias, definidas como las capacidades que las personas poseen para desempeñar una función productiva en escenarios laborales, al usar diferentes recursos bajo ciertas condiciones, que aseguran la calidad en el logro de los resultados (Chiavenato, 2002, p.23).

Las competencias de los trabajadores se manifiestan en tres niveles fundamentales: lo que se sabe (referido a las destrezas y habilidades relacionadas con el contenido de la tarea en general, el Saber), lo que se sabe hacer (referido al desempeño en un entorno laboral específico, el Saber Hacer) y el llamado Saber Estar referido a los aspectos más avanzados (motivación, sentido de pertenencia, iniciativa, autodisciplina...).

Los saberes hacer y estar están estrechamente vinculados con un segundo aspecto de vital importancia en los insumos de los procesos: el nivel en que esas competencias laborales son identificadas y utilizadas por la organización. En la medida en que los trabajadores se sientan reconocidos y debidamente utilizados, aportarán iniciativas, se sentirán involucrados, comprometidos y aumentará su satisfacción con el trabajo y con la organización.

Un tercer elemento que caracteriza los insumos organizacionales es el equipamiento que se utiliza en los procesos. Mientras más intensa sea la participación de los equipos en la obtención de un producto o la prestación de un servicio, más necesaria se hace la atención a los trabajadores de manera que mantengan su iniciativa, creatividad, pasión y entusiasmo.

Con el equipamiento se procesan de algún modo los materiales u objetos de trabajo. Son ellos los que van sufriendo un cambio cualitativo paulatino durante el proceso productivo, donde las competencias humanas propician la agregación de valor. Contablemente se presentan como activos circulantes porque entran y salen constantemente del proceso y trasladan su valor completamente, o en pocos ciclos productivos, al producto/servicio final.

Como materiales clasifican las materias primas, los semiproductos, partes y piezas, así como componentes completos que se incorporan al producto, los suministros energéticos de todo tipo, el agua 0 aire, y todo lo que de alguna manera se incorpore al producto/servicio final. También clasifican como materiales aquellos que faciliten el funcionamiento del equipamiento o el flujo de procesos, como los lubricantes, refrigerantes, accesorios, medios de protección, envases, embalajes, medios de manipulación o unitarización, entre otros.

La información es otro elemento que constituye insumo de los procesos. Hay información previa al 
proceso productivo o de prestación del servicio, referida a los clientes actuales o potenciales, los productos, servicios o soluciones integrales que desearían, las tendencias futuras de esos comportamientos, las exigencias y regulaciones del entorno, los proveedores actuales o potenciales, su comportamiento futuro, el comportamiento de la economía en general, y del mercado en particular, entre otros (Porter, 2010, p.11).

Se precisa también de información propia del proceso como las cantidades que hay que elaborar, los requisitos que deben cumplir, los materiales que se van a utilizar, sus características, los estándares de costos que se deben cuidar, las medidas que se deben tomar. Hay por último, información necesaria posterior al proceso como el cumplimiento del plazo de entrega pactado con el cliente, las opiniones expresadas sobre la calidad, precio y entrega de los productos o servicios, o las sugerencias que pueden contribuir a su mejora (Noori \& Radford, 1997, p.124).

\section{Procesos del sistema organizacional}

Durante los procesos de agregación de valor se cumple en buena medida la misión de las organizaciones. (OIT - CINTERFOR, 2014) Un significativo porcentaje de los recursos disponibles en la organización se consumen en dichos procesos, por lo que la medición de su utilización es un indicador de eficiencia de la gestión organizacional en su conjunto.

Todo proceso es eficiente cuando sus resultados son superiores en valor a sus insumos. Mientras la relación resultados/insumos, o de salidas/entradas se acerque más a 1, habrá más eficiencia en el proceso y la organización estará cumpliendo los requisitos mínimos para proyectarse hacia metas mayores.

En la gestión de operaciones, por tanto, el seguimiento de los indicadores de rendimiento ya sea de los hombres, los equipos o los materiales utilizados es de vital importancia.

Un segundo elemento de los procesos que es de interés para la gestión de operaciones es el tipo de proceso y su localización.
Los tipos de proceso pueden ser clasificados según la cantidad de unidades o volumen que se procesa de una vez o por el tamaño del lote. Asimismo, se pueden clasificar los procesos por su localización, por la distribución del equipamiento en el área de proceso, o por el tipo de modificación que ocurre en el producto final (Domínguez, 1995, p. 371).

Por el tamaño del lote, los procesos pueden clasificarse en: masivos, seriados y unitarios.

Un proceso masivo es característico de las producciones continuas que no se detienen, o lo hacen en situaciones excepcionales. En estos procesos no se puede determinar un lote productivo, porque no ocurren intervalos de paradas que permitan identificar principio o fin de un proceso. Se dice que en los procesos masivos el tamaño del lote tiende a infinito.

Un proceso es unitario cuando de cada ciclo productivo sale un producto diferente al anterior, por lo que un mismo equipamiento productivo permite la obtención de múltiples productos. En los procesos unitarios el tamaño del lote tiende a 1.

Un proceso es seriado cuando con el mismo equipamiento se procesan diferentes productos, con intervalos para hacer ajustes, que permitan acometer la nueva producción. Se diferencian tres niveles: grandes, medianas y pequeñas series.

La clasificación de la producción seriada en pequeña, mediana o gran serie depende del sector al que pertenezca la empresa que se estudie. En las empresas de la industria alimentaria, química ligera y otras similares, se producen tantos tipos de productos como el equipamiento tecnológico sea capaz de procesar.

La industria sidero-mecánica que fue el origen de estas clasificaciones incluye procesos siderúrgicos cuyo tamaño de lote es tan grande, que casi pudiera considerarse producción masiva. A esta rama de la industria también pertenece la producción de pequeños componentes metálicos de variadísimas formas, que convierten a la producción seriada en casi unitaria (Weihrich \& Koontz, 2004, p.83).

En la Tabla No. 1 se detallan las diferencias entre unas y otras 
Tabla 1.

\section{Comportamiento de los procesos seriados}

\begin{tabular}{|c|c|c|c|c|c|}
\hline $\begin{array}{c}\text { Tamaño de la } \\
\text { serie }\end{array}$ & Tamaño del lote & $\begin{array}{c}\text { Cantidad de } \\
\text { series }\end{array}$ & $\begin{array}{c}\text { Tiempo de } \\
\text { preparación del } \\
\text { proceso }\end{array}$ & $\begin{array}{c}\text { Duración total del } \\
\text { ciclo de proceso }\end{array}$ & $\begin{array}{c}\text { Tipo de } \\
\text { equipamiento }\end{array}$ \\
\hline Grandes series & Muy grande & Pocas & Largo & Largo & Especializado \\
\hline Medianas series & Medianas & Muchas & $\begin{array}{c}\text { Menor que en las } \\
\text { grandes series }\end{array}$ & Largo & $\begin{array}{c}\text { Universal y } \\
\text { especializado }\end{array}$ \\
\hline Pequeñas series & Pequeño o único & Muchas y diversas & Muy largo & $\begin{array}{c}\text { Relativamente } \\
\text { corto }\end{array}$ & Universal \\
\hline
\end{tabular}

Fuente: Elaboración propia

Según la posibilidad de movimiento de los objetos de trabajo, los procesos pueden clasificarse en: de localización única o electiva. Son procesos de localización única aquellos en que el objeto que se modifica no se transporta, sino que el equipamiento y los hombres se trasladan hacia el lugar para prestar el servicio.

Una parte significativa de las empresas que tienen un objeto de trabajo proveniente de la naturaleza, o que modifican el paisaje en beneficio humano, ejecutan procesos de localización única. Estos objetos de trabajo pueden ser vetas minerales, en el caso de empresas mineras; determinado gradiente de fertilidad, o condiciones climáticas que propicien un cultivo específico, para empresas agrícolas; o la construcción de un puente, un edificio o una autopista, en el caso de una empresa constructora. En todos estos casos se ejecuta el proceso solo donde se localiza el recurso que será objeto de trabajo.

La localización de los procesos y con ellos, de las empresas donde se ejecutan depende de múltiples factores: disponibilidad del talento humano para ejecutarlos, cercanía de los mercados, facilidades infraestructurales, regulaciones legales y otras muchas. De ahí que la localización electiva sea tan amplia que constituye en sí misma el contenido de estudio de muchas ramas del saber diferentes, que van desde la sociología hasta la geología.

Por la distribución del equipamiento productivo hay tres clasificaciones de procesos:
La distribución de un proceso es por producto, cuando la secuencia de operaciones tecnológicas a realizar para obtener un producto determina la secuencia de ubicación del equipamiento. Este es el caso de un sinnúmero de producciones masivas. La generación termoeléctrica de electricidad es un ejemplo típico de distribución por producto: siempre a la caldera de vapor le seguirá en el proceso una turbina, que estará acoplada a un generador eléctrico, que a su vez, estará seguido de una batería de transformadores.

Un proceso estará distribuido por procesos cuando el equipamiento se agrupa por similitud de las operaciones tecnológicas que se ejecutan con él. Así hay talleres de las industrias de conformación donde las máquinas están agrupadas por familias: tornos, taladros, pulidoras, soldadores...

Hay distribución mixta cuando el proceso productivo exige altos niveles de especialización en una fase del proceso, y altos niveles de eficiencia en otra. En esos casos se adopta la eficiente distribución por productos, en la fase en que la eficiencia es necesaria; y se acude a la distribución por procesos, en la fase donde la especialización es determinante.

Por último, pueden clasificarse los procesos por la manera en que se modifica el objeto de trabajo en: beneficiadores, convertidores y conformadores.

Procesos beneficiadores son todos los que se limitan a facilitar el posterior proceso de conversión. 
Incluyen los procesos de obtención de recursos naturales como la silvicultura, la minería, la pesca, la cosecha agrícola, y similares.

Son procesos convertidores aquellos que transforman las propiedades físico-químicas de los objetos de trabajo. Una manera sencilla de identificarlos es comparando las características de las materias primas que consumen y de los productos que entregan. La industria química es el ejemplo clásico para este tipo de procesos.

Los procesos conformadores no necesariamente cambian las propiedades físico-químicas, sino la apariencia del objeto de trabajo. La producción de artículos para el hogar, las confecciones textiles, la industria poligráfica, son ejemplos de procesos conformadores.

\section{Resultados o salidas del sistema organizacional}

Toda organización productiva o de servicios existe, porque sus productos o servicios satisfacen necesidades 0 expectativas de alguien. En el preciso momento en que el ciclo del capital se cierra, es decir, cuando un cliente paga el servicio o el producto que la organización le facilitó, es en ese momento en que se cumple la misión organizacional.

El ciclo de capital se describió como flujo financiero en la Figura No. 1. Se refiere al comportamiento que adoptan los recursos financieros durante el ciclo productivo.

La organización dispone de una cantidad determinada de dinero (D) con la que cuenta para cumplir su misión. Ese dinero (D) se invierte en la adquisición de mercancías (M) que son los recursos que precisa para ejecutar los procesos misionales, de apoyo y estratégicos. Esos recursos serían los insumos del proceso productivo o de servicios, según muestra la Figura No. 2.
Figura 2.

\section{Elementos de atención en la gestión de operaciones}

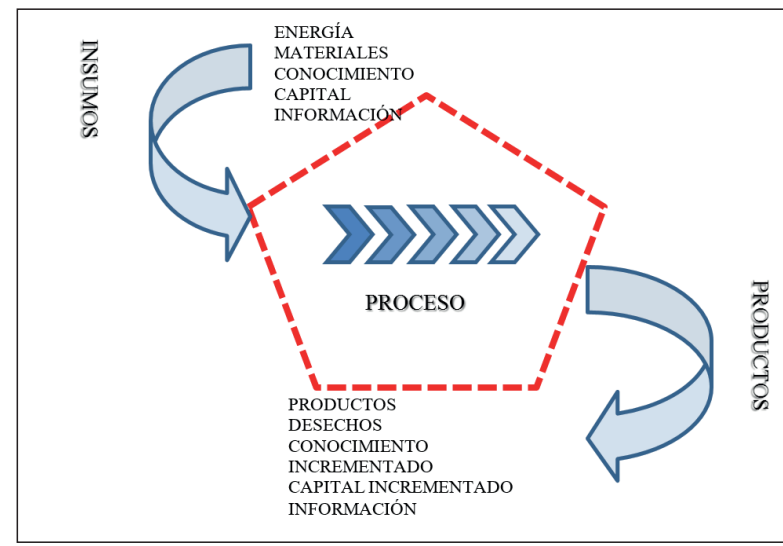

Fuente: Elaboración propia

Luego de ejecutado el proceso, se obtienen nuevos productos o servicios destinados a la venta, cuyo valor es cuantitativamente diferente y cualitativamente mayor que los insumos consumidos durante el proceso, por lo que se considera mercancía incrementada $\left(M^{\prime}\right)$ que al ser comercializada se transforma en dinero incrementado $\left(D^{\prime}\right)$.

Para que el ciclo del capital se cierre, es preciso que se vendan los productos o servicios por los clientes, y para ello, es necesario saber quiénes son, dónde están y cuáles son sus necesidades (Marx, 1998).

Saber quiénes son los clientes determina sobre los posibles precios de los productos o servicios, la mezcla que se haga, el diseño, las técnicas de mercadeo a utilizar, el momento y manera de hacer los lanzamientos de nuevos productos, entre otras decisiones táctico-operativas (y en muchos casos también estratégicas) futuras.

Dónde están los clientes determina la localización, y con ello, los planes de distribución, transporte y comercialización, así como las frecuencias y magnitudes de entrega. 
Las necesidades de los clientes están fuertemente relacionadas con los dos aspectos anteriores y dependen de un conjunto de factores socioeconómicos muy específicos de cada región (poder adquisitivo, salario medio, inflación, empleo, tradiciones, hábitos, costumbres, estructura etaria, nivel cultural, clima...) (Maynez, 2011).

De la misma forma constituyen aspectos a decidir las mezclas de productos/servicios que se conforman y sus adecuaciones para que sean del agrado de los clientes.

\section{Conclusión}

Las empresas están expuestas al nuevo reto de una competitividad en igualdad de condiciones son independencia de país o región, a causa del propio desarrollo tecnológico de la humanidad que facilita en intercambio informativo y las comunicaciones.

Considerar el impacto de la tecnología y la normalización determina la competitividad empresarial en los momentos actuales. Son las tecnologías de procesos las que han ido cambiando. Las sustituciones puntuales de equipamientos se han convertido en reposiciones totales de líneas de producción completas. El equipamiento se produce con altos estándares de fiabilidad, para un período definido de tiempo de explotación que los fabricantes cuidan mucho de declarar. La producción de componentes aislados de reposición para los equipos es cada vez menor $y$, a cambio, los gastos de mantenimiento crecen notablemente a costa de los elementos del costo vinculados al trabajo vivo. Una parte considerable del equipamiento productivo se adquiere incluyendo el mantenimiento especializado, brindado por el fabricante.

Esta igualdad de condiciones se manifiesta en un entorno normativo común a través de la familia de normas ISO, cuyo enfoque dirigido a procesos y no a funciones, impone nuevos paradigmas al ciclo cliente-proveedor.

\section{Referencias Bibliográficas}

Alford, B, Bangs, H y A. Hagemann (1953). Manual de la producción. México: Unión Tipográfica Editorial Iberoamericana

Andreu, V. (2013). Enfoque por procesos vs. enfoque por funciones. Obtenido de AMERICALEARNING\&MEDIA: http:// www.americalearningmedia.com / edicion-013/159-opinion/1405-gestion-por-procesosversus-gestion-por-funciones

Castillo Coto, A. L. (2015). Manual para la solución de problemas empresariales. Quito: La Huella. Colección Empresariales.

Castillo Coto, A. L. (01 de 2016). Curso de Gestión de Operaciones en Empresas de Servicios. Obtenido de Centro Virtual Universidad Metropolitana del Ecuador: http:// centro-virtual.umet.edu.ec/

Chiavenato, I. (2002). Administración en los nuevos tiempos. Bogota: McGraw Hill.

Domínguez Machuca, J. (1995). Dirección de operaciones. Madrid: McGraw Hill, 1era Ed.

ISO. (26 de 03 de 2015). Recuperado el 26 de 03 de 2015, de International Organization for Standardization: http:// www.iso.org/iso/home/news_index.htm

IS0/IEC 20000-3:2012. (2012). Part 3: Guidance on scope definition and applicability of ISO/IEC 20000-1. Information technology -- Service management. ISO.

Marx, K. (1998). El Capital. Tomo 1: El proceso de producción del capital. México: Siglo XXI.

Maynez Guaderrama, A. (2011). La transferencia de conocimiento organizacional como fuente de ventaja competitiva sostenible : modelo integrador de factores y estrategias. Recuperado el 06 de 02 de 2015, de OCLC - World Cat: http:// www.worldcat.org/title/transferencia-de-conocimientoorganizacional-como-fuente-de-ventaja-competitivasostenible-modelo-integrador-de-factores-y-estrategias/ oclc/857714349\&referer=brief_results

Noori, H \& R. Radford. (1997). Administración de operaciones y producción: calidad total y respuesta sensible. Bogotá: McGraw Hill - Interamericana.

OIT (2014). Recuperado el 01 de 03 de 2015, de Competencias laborales y formación profesional: http://www. oitcinterfor. org/competencias/inicio

Porter, M. (2010). Ventajas competitivas. Creación y sostenibilidad de un rendimiento superior. Madrid: Pirámide.

Weihrich, H \& H. Koontz. (2004). Administración. México: McGraw Hill. 